\title{
Properties of the Scattering Amplitude in the Renormalized Lee Model and in a Relativistic Generalization.
}

\author{
R. Collina \\ Istituto di Fisica dell' Unicersità - Firenze \\ Istituto Nazionale di Fisica Nucleare - Sezione di Firenze \\ G. LuzzatTo \\ Istituto di Fisica dell'Unicersità - Genov'a \\ Istituto Nazionale di Fisica Nucleare - Sezione di Genova \\ (Nuoro C'imento, $65 \mathrm{~A}, 277(1970)$ )
}

Two terms are missing in the equation on the lines 8 and 9 of page 293 . The correct form is

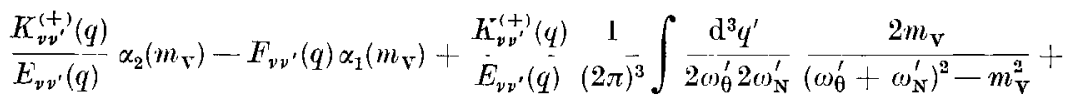

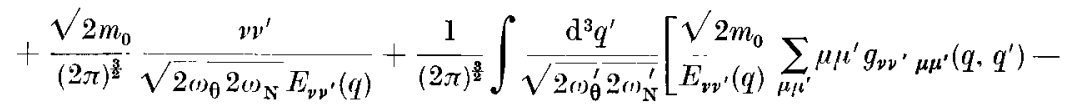

$$
\begin{aligned}
& \left.-\frac{1}{2(2 \pi)^{\frac{3}{2}} m_{\mathrm{V}}}-\frac{F_{\nu v^{\prime}}(q)}{\sqrt{2 \omega_{\theta}^{\prime} 2 \omega_{\mathrm{N}}^{\prime}}}\left(\frac{1}{\left(\omega_{\mathrm{N}}^{\prime}-\omega_{\theta}^{\prime}-m_{\mathrm{V}}\right)^{2}}+\frac{1}{\left(\omega_{\theta}^{\prime}-\omega_{\mathrm{N}}^{\prime}-m_{\mathrm{V}}\right)^{2}}\right)\right]=0 ；
\end{aligned}
$$

this boundary condition for the amplitudes, like the nonrelativisti one $\left(7^{n}\right)$, is not homogeneous. It derives from the homogeneous condition for the operators (lines 5 and 6 (if page 292) exactly in the same way as eq. $\left(7^{\prime \prime}\right)$ derives from eq. $\left(7^{\prime}\right)$.

Further, a trivial change has to be made in formula (17), and in other formulae on the same page 285, where the quantity $+i \varepsilon$, appearing as a consequence of the ontyoing wave condition, has to be cancelled from the denominators of the pole terms, surviving only in the integrals. 'The same cancellation of $t i \tau$ in the denominators of the pole terms has to be performed on page 294 for the relativistic case. 